\title{
Saturación del servicio de urgencias en la ciudad de Bogotá: una comprensión sistémica del problema
}

\author{
Overcrowding of emergency services in the city of Bogotá: \\ a systemic understanding of the problem
}

Juan Carlos Morales Piñero (https://orcid.org/0000-0003-2979-4839) ${ }^{1}$

Adriana Díaz (https://orcid.org/0000-0002-5316-432X) ${ }^{2}$

Juan Sebastián Martínez Vela (https://orcid.org/0000-0003-3913-2089)

${ }^{1}$ Programa de Ingeniería Industrial, Universidad Sergio Arboleda. Calle 74

14-14. 110221. Bogotá. Colombia.

juan.morales@usa.edu.co

${ }^{2}$ Departamento de

Ingeniería Industrial,

Pontificia Universidad

Javeriana. Bogotá Colombia.

\begin{abstract}
Emergency overcrowding is often associated with issues of inefficiency in care. However, this work focuses on another relevant aspect, namely care of non-urgent patients. In order to understand the dynamics associated with this aspect, a conceptual model of systems dynamics is constructed, based on the characterization of users and their decision criteria. A descriptive study was conducted based on a structured survey; additionally, binomial logistic regression and analysis of variance were used to estimate probability of emergency department visits and to define differences among users. The following criteria are relevant: service effectiveness, availability of medications and exams, and proximity to the emergency center. The waiting time is more significant for pediatric patients and the availability of medications and exams is also more prevalent for middle-income users; the latter attributes less importance to cost compared to low income. The model shows that resources create a virtuous or a vicious cycle of users waiting for care in both emergency and priority care (PC) centers. The proposal suggested is for segregation for pediatric users in PC centers and the promotion of user confidence in alternative options to emergency centers.
\end{abstract}

Key words Overcrowding, Emergency services, Social behavior, Understanding
Resumen La saturación de urgencias a menudo se asocia con ineficiencias en la atención. Sin embargo, este trabajo centra la mirada en otro aspecto relevante como es la asistencia de usuarios no urgentes. Para comprender las dinámicas asociadas a ello, se construye un modelo conceptual de dinámica de sistemas a partir de una caracterización de usuarios y de sus criterios de decisión. Se realizó un estudio descriptivo a partir de una encuesta estructurada, definiendo, a partir de una regresión logística binomial y un análisis de varianza, las diferencias entre usuarios y su probabilidad de asistencia a urgencias. Se encontraron como criterios relevantes: eficacia del servicio, disponibilidad de medicamentos y exámenes y cercanía del centro a la vivienda. El tiempo de espera es más significativo para pacientes pediátricos y la disponibilidad de medicamentos y exámenes lo es para usuarios de ingreso medio; estos últimos le dan menos importancia al costo comparados con los de ingreso bajo. El modelo muestra que los recursos generan un ciclo virtuoso o vicioso de usuarios en espera de atención tanto en urgencias como en atención prioritaria (AP). Se propone una segregación para usuarios pediátricos en la AP y fomentar la confianza de los usuarios en las opciones alternativas a los centros de urgencias.

Palabras clave Saturación, Urgencias, Comportamiento social, Comprensión 


\section{Introducción}

La saturación en los servicios de urgencias es un problema universal, y el debate sobre las causas que pueden explicar ese comportamiento y las posibles soluciones, sigue abierto ${ }^{1}$. Sin embargo, es necesario realizar investigaciones que aborden este problema y que, más allá de identificar causas, profundicen en su comprensión sistémica. Savigny y Adam $^{2}$ plantean que los sistemas de salud son complejos, de manera que para entender y trabajar con esta complejidad se requiere cambiar el paradigma de la aproximación reduccionista y lineal a un enfoque dinámico y holístico que considere las interrelaciones entre los componentes del sistema de salud, así como los intereses y perspectivas de los diferentes actores que hacen parte del sistema. La dinámica de sistemas es un método que soporta el aprendizaje sobre la complejidad dinámica y la resistencia a políticas presentes en un sistema social, de tal manera que se pueda diseñar políticas efectivas para intervenirlo. Múltiples trabajos, conceptuales o de simulación, han utilizado dinámica de sistemas para construir modelos que apoyen la definición de intervenciones a los sistemas de salud ${ }^{3-8}$.

En este sentido, se plantea que el sistema de urgencias puede clasificarse como un sistema social porque tanto el todo como las partes (p. ej., pacientes, empresas prestadoras de salud, trabajadores de la salud ) que lo constituyen tienen sus propios propósitos, a diferencia de otros tipos de sistemas como las máquinas, en los que ni el todo ni las partes tienen un propósito o preferencia $^{9}$. Los sistemas sociales son conducidos por las acciones y decisiones que toman los actores del sistema y su mutua interdependencia con el contexto en el que interactúan. Por consiguiente, el problema de saturación en urgencias es resultado de estas interacciones del sistema, de manera que las propuestas para rediseñarlo no deberían enfocarse en ciertos factores solamente, sino que deberían buscar cambiar o crear nuevas interacciones o reglas de decisión de sus actores.

La problemática objeto de estudio corresponde a la actual saturación que presentan las salas de urgencias de alta complejidad en la ciudad de Bogotá, Colombia. Esta situación ya ha sido documentada en un estudio anterior para Bogotá donde se analizaron las características y las capacidades de los Departamentos de Urgencias y que, llegó a la conclusión de que los servicios mantienen altos volúmenes de visitas, saturación de su capacidad $y$ largos tiempos de espera ${ }^{10}$.

El actual sistema de salud de Colombia se implementó a través de la Ley 100 de $1993^{11}$ y se basó en el modelo del Banco Mundial denominado Competencia Gerenciada. El modelo migró de una administración centralizada por parte del Estado, a una descentralización por funciones en instituciones independientes basadas en la competencia de mercado ${ }^{12}$. Se crearon dos regímenes de afiliación al sistema: el contributivo dirigido a personas con contrato laboral o trabajadores independientes que tienen capacidad financiera para aportar al sistema; y el subsidiado a quienes se les financian los servicios de salud ${ }^{13}$.

Los dos servicios principales en el sistema de salud a los cuales puede acudir el usuario que presenta una patología urgente o que requiere atención prioritaria (ser atendido en menos de 48 horas) son: las Unidades Básicas o Permanentes de Atención (UBA-UPA) y el centro de urgencias. Las UBA-UPA están pensadas para atender casos donde no está en riesgo la vida de las personas, por ello, estos centros cuentan típicamente con servicios de medicina general y si requiere de una atención inmediata, el paciente es remitido a urgencias. Cabe destacar que, si el usuario se encuentra en régimen contributivo, cada vez que haga uso de los servicios de las UBA-UPA, tales como consultas, exámenes, medicamentos, debe realizar un pago denominado "cuota moderadora".

Por el otro lado, a urgencias puede acudir un usuario sin cita previa, idealmente cuando su situación de salud presenta un peligro grave para la vida $^{14}$. Cuando se acude al servicio de urgencias con una situación muy grave (triage nivel I), la atención debe ser inmediata; si no, se realiza un filtro denominado "triage" donde se valora la gravedad de la situación del usuario. El triage por lo general es realizado por un profesional de enfermería; si su situación se considera grave (triage nivel II o III) se procede a la toma de exámenes y consulta con médicos y especialistas; por el contrario, si no es grave (triage nivel IV y V), el usuario puede no ser atendido y se le recomienda otro tipo de servicio ${ }^{15}$. A diferencia de los servicios en las UBA-UPA, el servicio prestado en urgencias (incluidas las consultas, exámenes, medicamentos) no tiene costo, a menos que sea usado para urgencias no vitales.

Para el caso del servicio de urgencias de Bogotá, Morales et al. ${ }^{16}$ afirman que existe una gran descoordinación entre los servicios de UBA-UPA y de atención en urgencias. De acuerdo con su análisis, los usuarios que son remitidos desde la UBA-UPA reciben el mismo trato que aquellos que llegan por cuenta propia, generando así un incentivo para que los usuarios decidan acudir directamente al servicio de urgencias. De igual forma, los autores evidenciaron que en promedio 
la atención en urgencias oscila entre seis y nueve horas, lo cual contrasta en gran medida con el tiempo promedio para obtener una cita en niveles básicos de atención como la medicina general que puede oscilar entre tres a seis, y con especialistas puede durar semanas. Igualmente, la diferencia en los costos de acceso entre los dos sistemas podría crear un incentivo para que los usuarios decidan acudir directamente a urgencias tal como se deduce del trabajo de Benach et al. ${ }^{17}$. En este caso es relevante determinar qué tanta importancia le da el usuario a este incentivo frente a otros criterios de análisis.

El servicio de urgencias puede comprenderse como un proceso de tipo entradas-procesos-salidas, de manera que el usuario que llega al servicio es la entrada más relevante mientras que la salida, es el usuario con un diagnóstico y tratamiento. Desde esta perspectiva, la saturación del servicio de urgencias, podría explicarse básicamente por tres motivos: sobrecarga de capacidad por el exceso de usuarios, ineficiencias en el proceso o una combinación de ambas ${ }^{18-21}$.

En este sentido, esta investigación se centra en analizar la gran afluencia de personas que asisten al servicio de urgencias, que conllevan a una sobrecarga de la entrada pues, tal como indican diversos estudios, muchos usuarios buscan atención urgente sin una causa justificada lo que contribuye en buena medida a esta sobrecarga ${ }^{22-25}$. El aumento generalizado del número de usuarios puede asociarse con tres tipos de variables: las que forman parte del ámbito de la persona (posición socioeconómica, antecedentes culturales, y aspectos demográficos); las que vienen dadas por los actores que planifican el acceso al sistema (disponibilidad del servicio, barreras a la entrada, incentivos, y mecanismos de control); y las variables no controladas (epidemias, afluencias cíclicas, desastres naturales $)^{26}$.

Con respecto al papel que juegan los actores que planifican los mecanismos de acceso al sistema, estos pueden generar incentivos o desincentivos para usar el servicio, dependiendo de la respuesta de los usuarios a sus decisiones. Igualmente, el comportamiento del usuario ante las políticas de acceso al sistema va a depender de las variables que forman parte de su ámbito. Por ejemplo, sería de esperar que una persona con alto poder adquisitivo prefiera pagar un poco más a cambio de mayor comodidad; o sería de esperar que una persona con bajo poder adquisitivo, tenga mayores problemas de salud que una con mejor poder. En este sentido, Braun et al. ${ }^{27}$ afirman que la demanda del servicio de urgencias es in- versamente proporcional al nivel socioeconómico del usuario, siendo esta una buena aproximación al grupo de variables que forman parte del ámbito de la persona. Se puede inferir que el usuario tenderá a elegir la opción que le ofrezca la mejor combinación de criterios posible para tomar la decisión de acceder al servicio de urgencias. Dentro de los posibles criterios que puede valorar el usuario al momento de decidir dónde acudir, se encuentran: la rapidez en la atención, la cercanía a su domicilio, la calidad de la atención, la reputación del centro médico y el costo del servicio ${ }^{22,26}$.

Con el fin de elaborar planes que conduzcan al usuario al uso correcto de los servicios de atención urgente, este trabajo pretendió realizar una caracterización de los usuarios del sistema de urgencias en la ciudad de Bogotá y con base en esto, construir un modelo conceptual que permitiera una comprensión sistémica de las dinámicas asociadas con el flujo de personas hacia el servicio de urgencias. El modelo permite comprender cómo las decisiones de actores del sistema de urgencias se relacionan y pueden estar generando dinámicas que refuerzan el problema de saturación del servicio, en lugar de solucionarlo. El modelo conceptual tiene como objetivo plantear una explicación endógena de la saturación de urgencias a partir de la interacción entre variables y actores que son representados en el modelo. Esta comprensión es de utilidad no solo para la ciudad de Bogotá, sino que puede ser usado como base para evaluar medidas que se han tomado en otros contextos para el mismo problema en los servicios de urgencias.

\section{Métodos}

El estudio se realizó a partir de una encuesta estructurada aplicada entre diciembre 2016 y enero 2017 (ver Cuadro 1). Se trata de un estudio explicativo que busca analizar los criterios que evalúa un usuario al momento de tomar la decisión de acudir a un centro de atención de urgencia y descartar la posibilidad de ir a una UBA-UPA. A partir de la información obtenida se aplicó un modelo de regresión logística binomial con el fin de determinar la probabilidad de asistencia de un usuario a urgencias y seguidamente se realizó un análisis de varianza (ANOVA) para determinar diferencias de comportamiento entre usuarios. Finalmente, desde un enfoque de dinámica de sistemas, se construyó un modelo conceptual para comprender las dinámicas que generan el alto flujo de personas al servicio de urgencias. 
Cuadro 1. Instrumento aplicado.

ENCUESTA ATENCIÓN URGENCIAS - CRITERIOS EN EL USO DE SERVICIOS DEL SISTEMA DE SALUD

Esta encuesta forma parte del proyecto de investigación

"Propuestas de mejora para el sistema general de seguridad social en salud" financiado por la Universidad Sergio Arboleda.

La información es anónima, y será usada exclusivamente con

fines académicos.

1. Datos sociodemográficos

\begin{tabular}{|l|l|}
\hline Edad & Localidad \\
\hline Sexo & Estrato \\
\hline
\end{tabular}

2. Tipo de paciente

¿Cuál es su parentesco con el paciente?

a) Padre o madre

b) Hermano que vive con el paciente

Qué tipo de paciente es?

c) Tío o abuelo que vive con el paciente

d) Otro: R/

3. Marque con una $\mathrm{X}_{\text {¿Qué tipo de afiliación }}$ tiene?

$\square$ Contributivo

$\square$ Subsidiado

$\square$ Vinculado

$\square$ No tengo (Vaya a P. 4)

5. ¿Cuál es su EPS?

6. ¿Cuál es el monto que usted cancela por

acceder a este servicio?

\begin{tabular}{|r|r|}
\hline Fecha & \\
\hline Hora & \\
\hline Código & \\
\hline
\end{tabular}

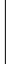

7. Para el manejo de una enfermedad calificando de 1 a 4 (siendo 4 mucha confianza) indique el nivel de confianza que tiene en los siguientes actores
a) Pediatrico
b) Adulto
c) Tercera edad

4. ¿Por qué NO está afiliado?

\begin{tabular}{|l|l|l|l|l|}
\hline Médico de la EPS & & & & \\
\hline Médico privado & & & & \\
\hline $\begin{array}{l}\text { Familiares y } \\
\text { amigos }\end{array}$ & & & & \\
\hline Droguería & & & & \\
\hline
\end{tabular}

8. ¿Fue remitido por el servicio de atención primaria?
a) $\$ 2.700$
b) $\$ 10.600$
c) $\$ 27.900$
d) Nada

9. ¿Por qué acudió a este centro? (Para cada afirmación indique la calificación que considere, siendo 4 totalmente de acuerdo y 1 totalmente en desacuerdo)

\begin{tabular}{|l|l|l|l|l|}
\hline $\begin{array}{l}\text { Porque se presentan bajos tiempo de espera en la } \\
\text { atención }\end{array}$ & $\begin{array}{c}\text { Totalmente de } \\
\text { acuerdo }\end{array}$ & $\begin{array}{c}\text { De } \\
\text { acuerdo }\end{array}$ & $\begin{array}{c}\text { En } \\
\text { desacuerdo }\end{array}$ & $\begin{array}{c}\text { Totalmente en } \\
\text { desacuerdo }\end{array}$ \\
\hline $\begin{array}{l}\text { Porque el servicio prestado por médicos y } \\
\text { enfermeras es acertado }\end{array}$ & & & & \\
\hline $\begin{array}{l}\text { Porque puede acceder luego a los medicamentos } \\
\text { y/o exámenes }\end{array}$ & & & & \\
\hline Porque el servicio es barato & & & & \\
\hline Porque el centro médico me queda cerca. & & & \\
\hline
\end{tabular}

continua

\section{Diseño y población}

La población de instituciones prestadoras de salud (IPS) con servicios de urgencias de nivel III en Bogotá es de 11 públicas y 10 privadas, ubicadas en 13 localidades ${ }^{28}$. De estas IPS se seleccionaron 12 centros por conveniencia y se aplicó una encuesta (ver Cuadro 1) de forma estratificada 
Cuadro 1. Instrumento aplicado.

\begin{tabular}{|c|c|}
\hline $\begin{array}{l}\text { 10. ¿Qué sintomatología o enfermedad está } \\
\text { presentando? }\end{array}$ & $\begin{array}{l}\square \text { Dolor de cabeza } \\
\square \text { Complicación respiratoria } \\
\square \text { Dolor abdominal } \\
\square \text { Dolor en las articulaciones } \\
\square \text { Diarrea, vómito } \\
\square \text { Fiebre alta } \\
\square \text { Otra Enfermedad:R/ }\end{array}$ \\
\hline $\begin{array}{l}\text { 11. ¿Ha acudido en los últimos tres meses a un } \\
\text { centro de urgencias? }\end{array}$ & $\begin{array}{l}\square \mathrm{Si} \\
\square \mathrm{No} \text { (Termine encuesta) }\end{array}$ \\
\hline \multicolumn{2}{|l|}{ 12. ¿Por cuál sintomatología acudió? } \\
\hline $\begin{array}{l}\text { 13. ¿Intento acudir a otro tipo de servicio como, } \\
\text { atención primaria, antes de ir a urgencias? }\end{array}$ & $\square$ No (Vaya directo a P. 15) \\
\hline \multicolumn{2}{|l|}{ 14. ¿Por qué no le solucionaron su problema? } \\
\hline \multicolumn{2}{|l|}{$\begin{array}{l}\text { 15. ¿Cuánto tiempo tuvo que esperar desde que } \\
\text { llegó hasta que fue atendido? (Minutos) }\end{array}$} \\
\hline $\begin{array}{l}\text { 16. ¿Realizó pago por exámenes, medicamentos u } \\
\text { otros servicios? }\end{array}$ & $\begin{array}{l}\square \text { Sí } \\
\square \text { No (Vaya directo a P. 18) } \\
\end{array}$ \\
\hline 17. El pago que realizó fue alto, normal o poco & $\begin{array}{l}\square \text { Alto } \\
\square \text { Normal } \\
\square \text { Poco }\end{array}$ \\
\hline $\begin{array}{l}\text { 18. ¿Le solucionaron, en urgencias, su problema } \\
\text { de salud con la primera visita? }\end{array}$ & $\square$ Sí $\square$ No ¿Por qué? \\
\hline 19. ¿Cuántas veces tuvo que volver? & $\mathrm{R} /$ \\
\hline
\end{tabular}

Fuente: Elaboración propia.

según tamaño de la población en diez localidades (Antonio Nariño, Barrios Unidos, Chapinero, Ciudad Bolívar, Engativá, Kennedy, Mártires, San Cristóbal, Suba, Usaquén), y en total se encuestó a 237 usuarios seleccionados de forma aleatoria en cada centro. De esta muestra, un $40.5 \%$ se realizó en instituciones privadas y el $59.5 \%$ a instituciones públicas.

Se realizó un muestreo estratificado tomando como criterio de homogeneidad los estratos socioeconómicos. La muestra se asignó teniendo en cuenta la proporcionalidad de la población respecto al estrato social utilizando los datos proporcionados por la Secretaría Distrital de Planeación. ${ }^{29}$ La muestra quedó definida así: uno centro para los estratos 0 y 1 (nivel socioeconómico más bajo), $11.09 \%$ de la población; cinco centros para el estrato 2, 39.36\% de la población; cuatro centros para el estrato $3,35.73 \%$ de la población; uno centro para el estrato $4,9.46 \%$ de la población y uno centro para los estratos 5 y $6,4.37 \%$ de la población.

\section{Variables del estudio}

En el estudio se tuvieron en cuenta variables del prestador del servicio (p. ej., cuota moderadora o calidad de la atención) y del usuario (p. ej., edad, sexo, estrato socioeconómico). Dado que se hicieron diferentes análisis sobre estos datos, se describen en el Cuadro 2 las variables utilizadas en cada análisis.

\section{Recolección, manejo y análisis de los datos}

Regresión logística: se aplicó un modelo de regresión logística binaria para estimar la probabilidad de que un usuario acuda directamente a urgencias $\mathrm{P}(\mathrm{Y}=1)$ o acuda antes a un UBA-UPA 1-P $(\mathrm{Y}=0)$ recogida por la variable "preferencia por urgencias". El modelo busca estimar los parámetros de una relación log lineal de la razón de probabilidades respecto a la combinación de las variables explicativas $\mathrm{X}$, obteniendo la estimación: $\ln \mathrm{P} / 1-\mathrm{P}=\beta_{0}+\mathrm{X}_{1} \beta_{1}+\ldots+\mathrm{X}_{\mathrm{n}} \beta_{\mathrm{n}}$; siendo $\mathrm{X}$ variables cuantitativas o cualitativas $(1,0)$ y $\beta$ el vector de los parámetros estimados por máxima 
Cuadro 2. Variables del estudio por tipo de análisis

\begin{tabular}{|c|c|c|c|}
\hline Variables del estudio $^{\text {a }}$ & $\begin{array}{c}\text { Regresión } \\
\text { logística }\end{array}$ & ANOVA & $\begin{array}{c}\text { Dinámica } \\
\text { de sistemas }\end{array}$ \\
\hline Sexo del paciente (1): "hombre" o "mujer". & $\mathrm{X}$ & & \\
\hline Edad del paciente (1): variable cuantitativa continua. & $\mathrm{X}$ & & \\
\hline $\begin{array}{l}\text { Estrato de la vivienda (1): es el mecanismo que clasifica la población con } \\
\text { características socioeconómicas similares en diferentes grupos o estratos } \\
\text { de acuerdo con la ubicación de la vivienda. Puede tomar valor de } 1 \text { a } 6 \text {, } \\
\text { siendo } 6 \text { el estrato con mayores recursos económicos. }\end{array}$ & & $\mathrm{X}$ & \\
\hline $\begin{array}{l}\text { Tipo de paciente (2): clasificación del paciente según su edad. Categoría: } \\
\text { pediátricos (de } 0 \text { a } 15 \text { años), adultos (de } 16 \text { a } 50 \text { años), tercera edad (de } \\
50 \text { años en adelante). }\end{array}$ & & $\mathrm{X}$ & \\
\hline $\begin{array}{l}\text { Régimen de afiliación (3): indica la modalidad de vinculación del usuario } \\
\text { al Sistema de Salud: subsidiado (el Estado cubre los costos), contributivo } \\
\text { (el usuario o un familiar pagan por su afiliación), y no afiliado. }\end{array}$ & $\mathrm{X}$ & $\mathrm{X}$ & \\
\hline $\begin{array}{l}\text { Nivel de confianza por tipo de agente (7): evalúa la confianza que tiene el } \\
\text { usuario de urgencias para el manejo de la enfermedad en los siguientes } \\
\text { actores: } \\
\text { a. Médico de los UBA-UPA } \\
\text { b. Médico privado } \\
\text { c. Familiares y amigos } \\
\text { d. Farmaceuta } \\
\text { Escala: } 4 \text { ) mucha confianza, 3) algo de confianza, 2) poca confianza, 1) } \\
\text { nada de confianza. }\end{array}$ & $\begin{array}{l}\text { a. X } \\
\text { b. X } \\
\text { c. X } \\
\text { d. X }\end{array}$ & & \\
\hline $\begin{array}{l}\text { Preferencia por urgencias (8): define si el usuario ante su patología acude } \\
\text { directamente a urgencias sin ir a un UBA-UPA. Categoría: "si" o "no" }\end{array}$ & $\mathrm{X}$ & & \\
\hline $\begin{array}{l}\text { Criterios para asistir a urgencias (9): define el grado de conformidad del } \\
\text { usuario respecto a los motivos que tuvo en cuenta para acudir a urgencias } \\
\text { a. Bajo tiempo de espera } \\
\text { b. Atención acertada } \\
\text { c. Disponibilidad de medicamentos y exámenes } \\
\text { d. Bajo costo del servicio } \\
\text { e. Cercanía a la vivienda } \\
\text { Escala: } 4 \text { ) totalmente de acuerdo, 3) de acuerdo, 2) en desacuerdo y 1) } \\
\text { totalmente en desacuerdo. }\end{array}$ & & $\begin{array}{l}\text { a. X } \\
\text { b. X } \\
\text { c. X } \\
\text { d. X } \\
\text { e. X }\end{array}$ & $\begin{array}{l}\text { a. } X \\
\text { b. } X \\
\text { c. X } \\
\text { d. X } \\
\text { e. X }\end{array}$ \\
\hline $\begin{array}{l}\text { Recurrentes en urgencias (11): Usuarios en urgencias que han asistido al } \\
\text { servicio en los últimos tres meses. Categoría: "si" o "no". }\end{array}$ & $\mathrm{X}$ & & \\
\hline $\begin{array}{l}\text { Presión para mejorar servicios urgencias: captura la presión que ejercen } \\
\text { los ciudadanos sobre el gobierno de la ciudad demandando mejoras en el } \\
\text { servicio de urgencias ante el problema de la saturación. }\end{array}$ & & & $\mathrm{X}$ \\
\hline $\begin{array}{l}\text { Usuarios Remitidos de UBA-UPA a urgencias: indica si el paciente fue } \\
\text { remitido al centro de urgencias por un UBA-UPA debido a la gravedad de } \\
\text { la patología o que requiere del concepto de un médico especialista o de } \\
\text { exámenes no disponibles en los UBA-UPA. }\end{array}$ & & & $X$ \\
\hline $\begin{array}{l}\text { Tiempo de espera para ser atendidos urgencias: minutos que tuvo que } \\
\text { esperar el usuario desde que llegó a urgencias hasta que fue atendido. }\end{array}$ & & & $\mathrm{X}$ \\
\hline $\begin{array}{l}\text { Población con enfermedad urgente (PEU): representa los habitantes de } \\
\text { Bogotá que presentan una patología que requiere atención urgente. }\end{array}$ & & & $\mathrm{X}$ \\
\hline $\begin{array}{l}\text { Población con enfermedad prioritaria no urgente (PEPR): usuarios que } \\
\text { presentan una enfermedad que no pone en riesgo su vida pero que debe } \\
\text { ser atendida en menos de } 48 \text { horas. }\end{array}$ & & & $\mathrm{X}$ \\
\hline $\begin{array}{l}\text { Usuarios en espera de atención en urgencias: personas esperando ser } \\
\text { atendidas. En este punto se encuentra un triage donde no se atiende la } \\
\text { condición de salud de los usuarios, sino que se valora y clasifica según la } \\
\text { gravedad de la enfermedad. }\end{array}$ & & & $\mathrm{X}$ \\
\hline $\begin{array}{l}\text { Usuarios que se van sin atención: personas que dejan el servicio sin } \\
\text { recibir atención oportuna }\end{array}$ & & & $\mathrm{X}$ \\
\hline
\end{tabular}


Cuadro 2. Variables del estudio por tipo de análisis

\begin{tabular}{|c|c|c|c|}
\hline Variables del estudio ${ }^{a}$ & $\begin{array}{c}\text { Regresión } \\
\text { logística }\end{array}$ & ANOVA & $\begin{array}{l}\text { Dinámica } \\
\text { de sistemas }\end{array}$ \\
\hline $\begin{array}{l}\text { Recursos disponibles en urgencias: se refiere a distintos tipos de recursos } \\
\text { que el sistema requiere para garantizar la atención de los pacientes; } \\
\text { incluye infraestructura, personal médico, equipos médicos y técnicos, etc. }\end{array}$ & & & $\mathrm{X}$ \\
\hline $\begin{array}{l}\text { Tiempo de espera para ser atendidos en UBA-UPA: tiempo que percibe el } \\
\text { usuario deberá esperar hasta ser atendido. Típicamente debe acercarse a } \\
\text { un centro que ofrezca este servicio en donde se le asigna una cita. }\end{array}$ & & & $\mathrm{X}$ \\
\hline
\end{tabular}

${ }^{\text {a }}$ Si la variable utiliza información del instrumento mostrado en el Cuadro 1, la pregunta que soporta la información está entre paréntesis después del nombre de la variable.

Fuente: Elaboración propia.

verosimitud. Las variables explicativas del modelo son: $\mathrm{X}_{1}$ sexo (1 hombre, 0 mujer), $\mathrm{X}_{2}$ recurrentes en urgencias ( 1 sí, 0 no), $X_{3}$ régimen de afiliación convertida en binaria (¿afiliado a régimen contributivo? 1 sí, 0 no), $\mathrm{X}_{4}$ edad del paciente (cuantitativa), $\mathrm{X}_{5}$ nivel de confianza en médicos de la EPS convertida en binaria (¿Confía en médicos de la EPS? 1 Sí (agrupa las escalas 4 y 3), 0 No (agrupa las escalas 2 y 1 ), $\mathrm{X}_{6}$ nivel de confianza en farmaceuta convertida en binaria ( $¿$ Confía en farmaceuta? 1 Sí (agrupa las escalas 4 y 3), 0 No (agrupa las escalas 2 y 1). Para correr el modelo se usó el software IBM SPSS Statistics versión 24.

ANOVA: para determinar si existían diferencias significativas en los criterios que valora el usuario que tiene preferencia por urgencias se corrió un ANOVA, cruzando las cinco categorías de la variable "criterios para asistir a urgencias" que ya segrega el subgrupo que respondieron "no" a la variable "preferencia por urgencias". Se aplicó el ANOVA a esta variable por estrato socioeconómico, tipo de paciente y régimen de afiliación, utilizando el Test de Levene para verificar la homogeneidad de las varianzas y la prueba post hoc de Scheffe asumiendo como diferencias significativas $P$-value iguales o menores al 0.05. Se utilizó el software IBM SPSS Statistics versión 24.

Diagrama de ciclos causales: a partir de información y análisis realizados en las etapas anteriores y de literatura sobre el problema, se construyó un diagrama de ciclos causales (DCC) que es una herramienta de modelación que permite representar la estructura de un sistema complejo, como el de salud ${ }^{30}$. Este DCC permite visualizar cómo se relacionan las variables en el sistema de urgencias y, además comprender las dinámicas de realimentación existentes que pueden estar regulando o amplificando la congestión del sistema. Los DCC proponen una aproximación diferente a las situaciones problemáticas que no se concentra en encontrar y atacar causas, sino que plantea que esas situaciones son resultado de las interacciones y realimentaciones internas del sistema ${ }^{30}$. Comprender esta estructura es central para definir intervenciones que sean realmente efectivas. Un DCC se compone de variables conectadas por flechas que representan la influencia causal entre variables. Estos enlaces causales tienen asociada una polaridad positiva $(+)$ o negativa (-), que indica la dirección del cambio que provoca una variable sobre la otra (ver Figura 1); una polaridad + indica que el cambio será en la misma dirección (p. ej., si los nacimientos aumentan, la población aumenta), y la polaridad - indica que el cambio será en dirección opuesta (p. ej., si hay más muertes, la población disminuye; si la esperanza de vida baja, las muertes se incrementan). A partir de las relaciones causales se identifican ciclos de realimentación positivos o de refuerzo (+) o ciclos de realimentación negativa o de balance (-) (ver Figura 1).

Los ciclos positivos pueden ser virtuosos o viciosos al reforzar que un comportamiento bueno o malo tome cada vez más fuerza, mientras que los de balance tratan de regular el sistema y que tienda al equilibrio. En el ejemplo de la Figura 1 la población se ve reforzada por los nacimientos y regulada por las muertes; en el caso de que no existiera el ciclo de balance de muertes, el ciclo de refuerzo podría generar que la población creciera infinitamente y cada vez más rápido, sin embargo, esto no ocurre ya que de las muertes limitan ese crecimiento.

Para el planteamiento de la dinámica del sistema de salud, se utilizó el software Vensim PLE.

El protocolo de la investigación fue aprobado por el Comité de Ética en Investigación de la Universidad. 


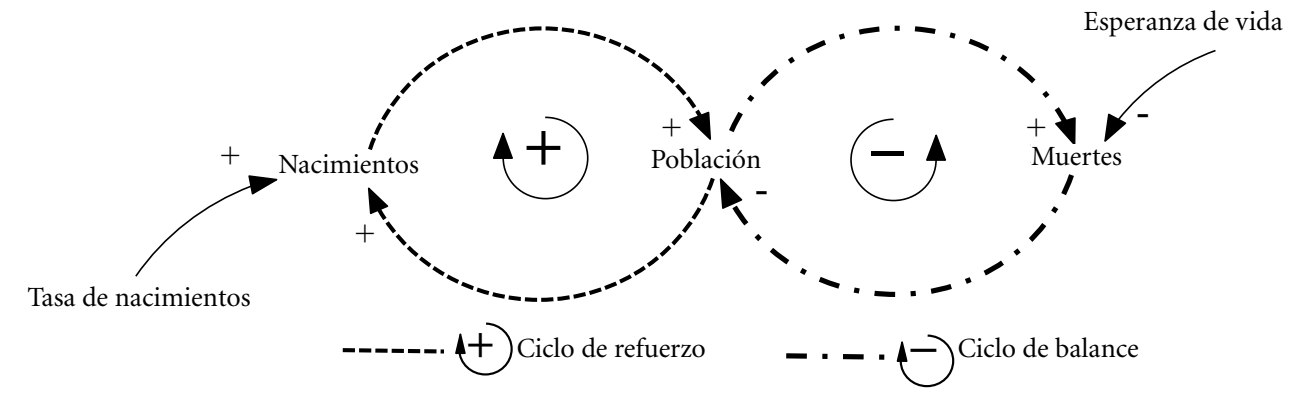

Figura 1. Ejemplo de relaciones causales y ciclos de realimentación.

Fuente: Elaboración propia.

\section{Resultados}

De la encuesta aplicada (ver Cuadro 1), se destacan los resultados que evalúan los criterios que tuvieron los usuarios para asistir al servicio de urgencias. Se encontró que cuando el usuario presenta alguna patología, tiende a percibir con mucha confianza la opinión que puedan ofrecerle los médicos y con poca confianza la opinión de los familiares y farmaceutas (ver Tabla 1). Al descartar aquellos usuarios remitidos por los UBAUPA (segregados ya por la variable "Criterios para asistir a urgencias"), ya que por lo general en estos casos la persona no tiene criterio de decisión propio, e indagar en los criterios evaluados por quienes tienen preferencias por urgencias, encontramos que los usuarios tienen un alto grado de conformidad con la eficacia del servicio (atención acertada $58.97 \%$ ), con la disponibilidad de medicamentos y exámenes $48.60 \%$ y con la cercanía del centro a su vivienda $56.59 \%$ (ver Tabla 1).

\section{ANOVA por criterios de usuarios que tienen preferencia por urgencias}

El análisis se centró en evaluar las diferencias de criterio que existen por tipos de usuarios que los hacen tener preferencia por urgencias (ver Tabla 2). Se determinó que existen diferencias significativas por tipo de paciente respecto al tiempo que están dispuestos a esperar para la atención ( $p$-value 0.009). El paciente pediátrico le da mayor importancia al bajo tiempo de espera respecto a los pacientes adultos y de tercera edad. Asimismo, se observa que al momento de acudir a urgencias la disponibilidad de medicamentos y exámenes es significativamente más importante para los usuarios afiliados al régimen contributivo ( $p$-value 0.000$)$ y para los usuarios de clase media (estrato 3) ( $p$-value 0.001). De igual forma, los usuarios de clase media le dan menos importancia al costo del servicio que los usuarios de clase baja ( $p$-value 0.009).

Esto indica que para usuarios de clase media es más importante disponer de medicamentos y exámenes que el costo del servicio. Por su parte, para los usuarios de clase baja el costo del servicio es mucho más importante que la disponibilidad de medicamentos y exámenes. Finalmente, es de resaltar la homogeneidad que se observa en la valoración que reciben los criterios de atención acertada y la cercanía a la su vivienda, lo que indica que independientemente del sexo, estrato, tipo de paciente y régimen, para los usuarios éstos son criterios relevantes para preferir el servicio de urgencias.

\section{Regresión logística}

Los resultados de la regresión logística indican que el modelo es adecuado (prueba de ómnibus p-value 0.001; prueba de Hosmer y Lemeshow $p$-value 0.167 ) y predijo correctamente el $75.3 \%$ de los datos. Como resultados significativos se destaca que los usuarios de sexo masculino tienen mayor probabilidad de asistir a urgencias que las mujeres (odds ratio 2.482; p-value 0.009). Asimismo, el haber asistido en los últimos tres meses a urgencias disminuye la probabilidad de regresar a la mitad (odds ratio 0.562; p-value 0.084). Este resultado puede deberse a lo acertada que sea la atención. Por otra parte, se destaca que un au- 
Tabla 1. Análisis descriptivo de los datos ${ }^{\mathrm{a}}$.

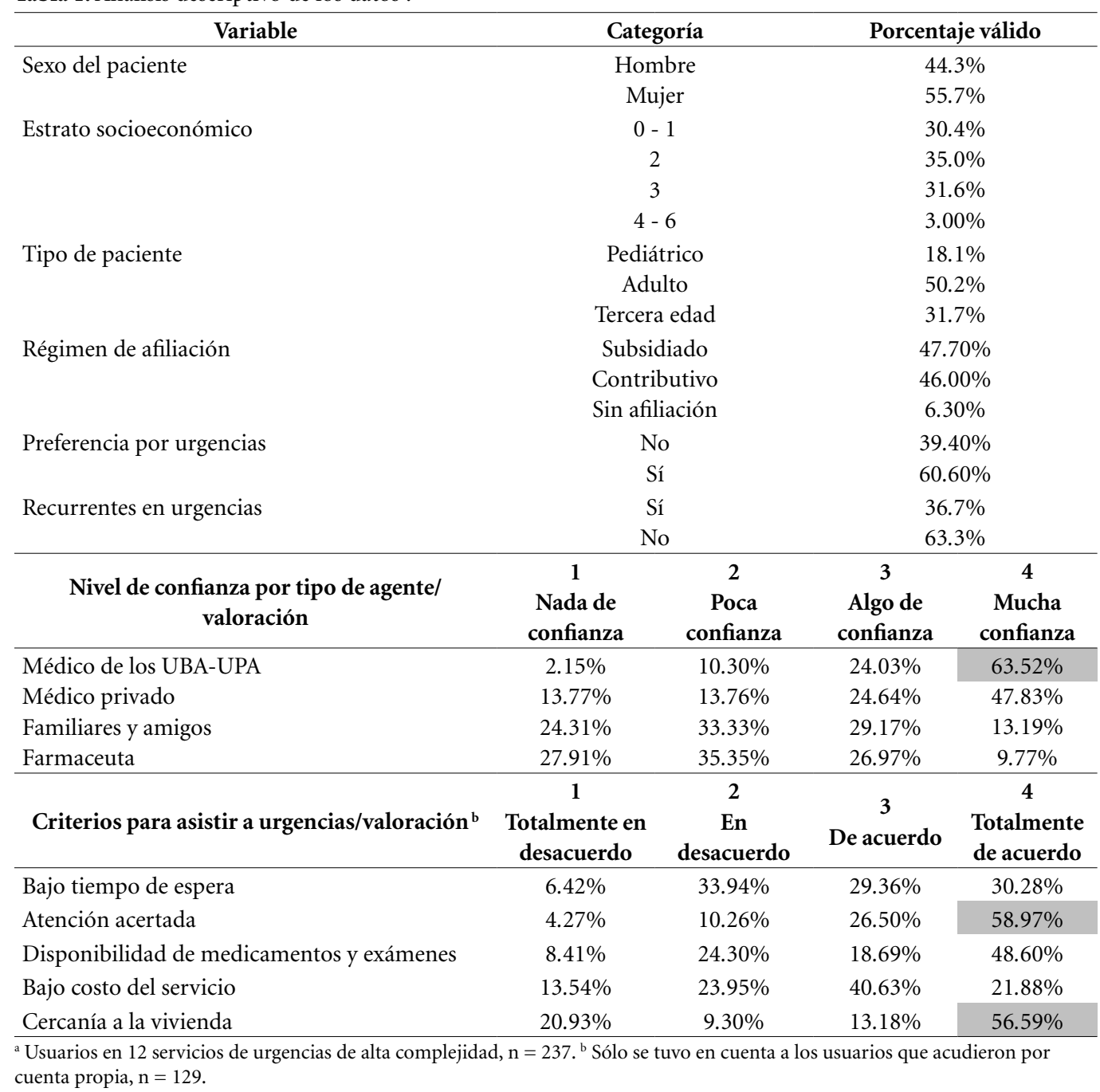

Fuente: Elaboración propia.

mento de la confianza de los usuarios en los médicos de los UBA-UPA disminuye la probabilidad de acudir a urgencias (odds ratio 0.338 ; p-value 0.066). Aunque el resto de variables no resultan significativas, se destaca que los usuarios que confían en la opinión de los farmaceutas aumentan la probabilidad de acudir a urgencias (odds ratio 1.758; p-value 0.105). Esto podría indicar que la orientación que reciben de los farmaceutas no resulta efectiva en la solución de la patología.

\section{Dinámicas relacionadas con el sistema de urgencias}

En la Figura 2 se presenta el modelo conceptual construido, en el que se plasman las relaciones entre variables y la decisión de ir a urgencias con base en lo encontrado en el análisis anterior y literatura en el tema. Además de esto, el modelo es valioso pues permite comprender cómo el problema de saturación surge de las dinámicas - de refuerzo y balance -que aparecen a partir de estas relaciones, y que no sólo dependen de decisiones de los usuarios sino también de los tomadores de decisiones que tratan de mejorar el servicio. Este modelo conceptual puede ser utilizado como base para la comprensión del problema de saturación en contextos diferentes a Bogotá y Colombia.

Una variable central en el modelo es "Personas enfermas que van a urgencias", que depende por un lado de las personas que presentan una patología urgente o prioritaria y, por otro lado, de variables asociadas a los centros de atención que podrían atender estas patologías. Lo primero se presenta en el modelo con dos variables: la Población con enfermedad prioritaria no urgente 
Tabla 2. Resultados del ANOVA por criterios para acudir a urgencias.

\begin{tabular}{|c|c|c|c|c|c|c|c|c|c|c|c|}
\hline \multirow{2}{*}{\multicolumn{2}{|c|}{$\begin{array}{l}\text { Variables de contraste } \\
\text { Media }\end{array}$}} & \multicolumn{2}{|c|}{$\begin{array}{l}\text { Bajo tiempo } \\
\text { de espera }\end{array}$} & \multicolumn{2}{|c|}{$\begin{array}{l}\text { Atención } \\
\text { acertada }\end{array}$} & \multicolumn{2}{|c|}{$\begin{array}{c}\text { Disponibilidad } \\
\text { de }\end{array}$} & \multicolumn{2}{|c|}{$\begin{array}{l}\text { Bajo costo } \\
\text { del servicio }\end{array}$} & \multicolumn{2}{|c|}{$\begin{array}{c}\text { Cercanía a la } \\
\text { vivienda }\end{array}$} \\
\hline & & $\begin{array}{c}\text { p- } \\
\text { valor }\end{array}$ & Media & $\begin{array}{c}\text { p- } \\
\text { valor }\end{array}$ & Media & $\begin{array}{c}\text { p- } \\
\text { valor }\end{array}$ & Media & $\begin{array}{c}\text { p- } \\
\text { valor }\end{array}$ & Media & $\begin{array}{c}\text { p- } \\
\text { valor }\end{array}$ & \\
\hline \multirow[t]{2}{*}{ Sexo } & Hombre & 2.77 & 0.392 & 3.29 & 0.546 & 2.83 & 0.321 & 2.68 & 0.216 & 2.93 & 0.854 \\
\hline & Mujer & 2.61 & & 3.18 & & 3.04 & & 2.42 & & 2.89 & \\
\hline \multirow{4}{*}{$\begin{array}{l}\text { Estrato } \\
\text { socioeconómico }\end{array}$} & $0-1$ & 2.66 & 0.564 & 3.42 & 0.991 & 2.41 & 0.001 & 3.24 & 0.009 & 3.20 & 0.403 \\
\hline & 2 & 2.98 & & 3.39 & & 3.33 & & 2.61 & & 2.82 & \\
\hline & 3 & 2.83 & & 3.43 & & 3.29 & & 2.43 & & 3.05 & \\
\hline & $4-6$ & 3.00 & & 3.50 & & 3.50 & & 3.00 & & 3.50 & \\
\hline \multirow{3}{*}{$\begin{array}{l}\text { Tipo de } \\
\text { paciente }\end{array}$} & Pediátrico & 2.25 & 0.009 & 3.58 & 0.607 & 3.06 & 0.824 & 3.00 & 0.199 & 3.05 & 0.987 \\
\hline & Adulto & 2.84 & & 3.37 & & 3.03 & & 2.57 & & 3.07 & \\
\hline & Tercera edad & 3.14 & & 3.36 & & 3.18 & & 2.87 & & 3.03 & \\
\hline \multirow{3}{*}{$\begin{array}{l}\text { Régimen de } \\
\text { afiliación }\end{array}$} & Contributivo & 2.76 & 0.108 & 3.45 & 0.095 & 3.37 & 0.000 & 2.31 & 0.087 & 2.81 & 0.196 \\
\hline & Subsidiado & 2.52 & & 3.03 & & 2.40 & & 2.78 & & 2.91 & \\
\hline & No afiliado & 3.15 & & 3.17 & & 3.25 & & 2.60 & & 3.50 & \\
\hline
\end{tabular}

Fuente: Elaboración propia.

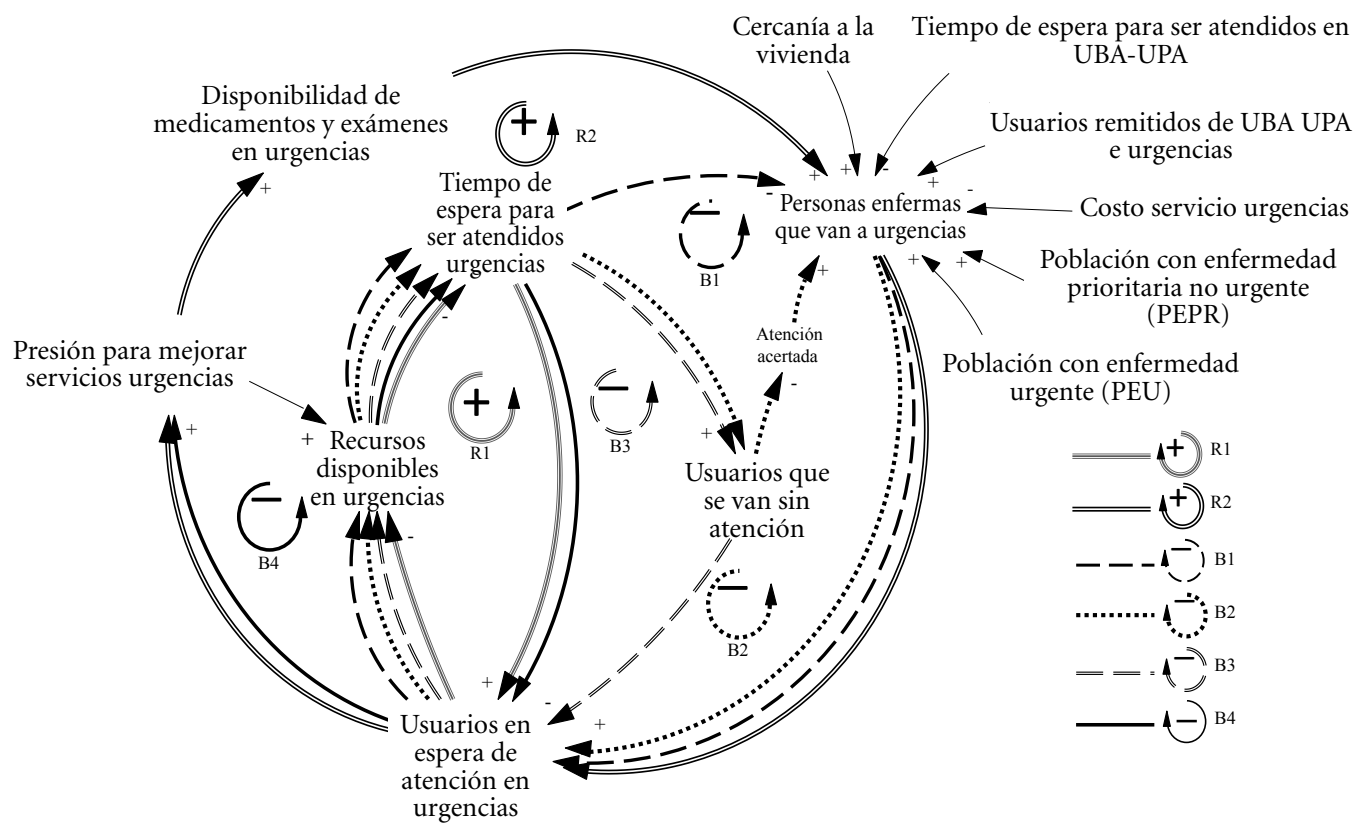

Figura 2. Dinámicas de personas esperando atención en urgencias.

Fuente: Elaboración propia.

(PEPR) que captura aquellas personas que deberían ir a los UBA-UPA y la Población con enfermedad urgente (PEU) que representa las personas que sí requieren atención en un centro de urgencias. En cuanto a las variables de los centros de atención que influyen en la decisión de las poblaciones (PEPR y PEU) de ir a urgencias, el modelo plantea cinco variables asociadas con el servicio de urgencias y dos con el servicio en los UPAUPA. Las asociadas con urgencias son: 1) el tiem- 
po de espera para ser atendidos, 2) la percepción de una atención acertada, 3) la disponibilidad de medicamentos y exámenes, 4) la cercanía a la vivienda y 5) el costo del servicio. Las dos variables relacionadas con la atención en los UBA-UPA son 6) el tiempo de espera para ser atendidos en un UBA- UPA ya que en la medida que este crece, las personas prefieren asistir a urgencias directamente; y 7) los pacientes remitidos a urgencias desde los UBA-UPA. El modelo conceptual que se construyó muestra cómo algunas de estas variables están interrelacionadas y hacen parte de ciclos que refuerzan o regulan la problemática de la saturación de los servicios de urgencias. A continuación, se explica en detalle estos ciclos y su función en el sistema.

El ciclo de balance B1 representa la regulación que ejercen los tiempos de espera sobre los usuarios que van a urgencias ante una enfermedad; Así, entre más personas enfermas van a urgencias, menos recursos disponibles hay en este servicio y por consiguiente mayores tiempos de espera. Esto último termina disminuyendo las personas que ante una enfermedad deciden ir a urgencias. Otro ciclo que balancea esta última variable es el B2 que incluye la percepción de los pacientes sobre la atención. El ciclo B2 muestra que al crecer el número de usuarios esperando ser atendidos se disminuyen los recursos disponibles, lo cual genera un incremento en los tiempos de espera de manera que hay usuarios que deciden irse sin recibir atención. Esta fuga de usuarios termina afectando negativamente la percepción de qué tan acertada es la atención en urgencias, regulando así la cantidad de personas enfermas que deciden ir a estos centros. Aunque este ciclo B2 comprende una regulación de la problemática, no es una dinámica muy deseada ya que implica que las personas se vayan sin atención.

El tiempo de espera en urgencias es una variable importante en el problema de la saturación de urgencias. El ciclo R1 define que este tiempo de espera es reforzado por los usuarios en urgencias; esto se debe a que los usuarios esperando atención implican menos recursos disponibles en urgencias (p. ej, sillas o camillas, enfermeras) y la disminución en estos recursos refuerza el incremento de los tiempos que los usuarios deben esperar para ser atendidos. También existe el ciclo B3 que frena el incremento de estos tiempos; sin embargo, similar a lo que pasa con el ciclo B2, no es una dinámica que deba ser promovida. El ciclo B3 captura el hecho que los usuarios que se van sin atención, tal como lo plantea Higginson ${ }^{31}$, liberan recursos y esto disminuye los tiempos de atención; no obstante, los pacientes salen sin un dictamen médico, es decir que el sistema no cumple con su misión.

La saturación de urgencias, representada en los usuarios en espera de atención, genera presiones para mejorar el servicio en estos centros. El modelo plantea que estas presiones ocasionan una mejora tanto en los recursos disponibles en urgencias como en la disponibilidad de medicamentos y exámenes; lo anterior crea dos ciclos: B4 y R2. El ciclo B4 muestra el efecto regulador que se busca con las presiones; así, a mayor presión, se logra frenar el crecimiento del tiempo de espera ya que hay más recursos disponibles. Por otro lado, el ciclo R2 puede estar ocasionando un comportamiento contraintuitivo pues, en lugar de mejorar la situación la pueden empeorar. Ante el alto número de personas esperando atención, hay presión para que se mejore el servicio y una de las cosas que se hace es aumentar la disponibilidad de medicamentos y exámenes en los centros de atención de urgencias. El ciclo R2 muestra que al aumentar esta disponibilidad, es más atractivo para las personas acudir directamente a urgencias ante una enfermedad, lo que termina reforzando el crecimiento de usuarios en urgencias.

\section{Discusión}

El modelo conceptual construido permite comprender cómo la decisión de los usuarios de ir a urgencias ante una enfermedad, no depende solamente de factores a nivel del individuo, sino que puede verse reforzada o regulada por dinámicas que resultan de las decisiones de los usuarios, pero también de otros stakeholders que tratan de mejorar el servicio de urgencias. Es para estos últimos, en especial, para quienes modelos como el construido tienen gran valor ya que permiten tener una visión holística del sistema y de cómo ciertas decisiones o partes se relacionan para generar el comportamiento observado.

Aunque el modelo es construido a partir de los datos recolectados para la ciudad de Bogotá, puede ser de gran utilidad en otros contextos. Con el modelo se plantea una manera diferente de pensar y de abordar el problema de saturación de urgencias. De hecho, como lo plantea Vermaak ${ }^{32}$, un modelo conceptual es una herramienta por sí sola muy útil al abordar sistemas complejos ya que permite hacer visibles mecanismos de realimentación que típicamente son invisibles a primera vista, pues las causas pueden estar distantes o desconectadas de las consecuencias o puede haber efectos retardados 
entre unos y otros. Como consecuencia, los tomadores de decisión enfrentan ciertas limitaciones cognitivas al tratar de definir soluciones donde las más obvias a menudo terminan agravando el problema, o la organización rechaza aquella solución que es factible. La necesidad de un pensamiento sistémico en el estudio de los sistemas de salud también ha sido planteada desde la Organización Mundial de la Salud con el trabajo de Savigny y Adam $^{2}$, quienes presentan las ventajas de una perspectiva sistémica en la comprensión de los sistemas de salud, así como en el diseño de intervenciones para estos; de hecho lo plantean como "un componente imprescindible para fortalecer el desarrollo general del sector de la salud en los países de bajos y medianos ingresos". En ese sentido, el modelo construido plantea una conceptualización sistémica de la afluencia de usuarios al servicio de urgencias; conceptualización que plantea estructuras de realimentación relacionadas con decisiones de diferentes actores del sistema.

Disminuir los tiempos de espera en urgencias es un problema que ha sido abordado por académicos alrededor del mundo; varios de ellos enfocados en optimizar el uso de los recursos como camas, y personal médico. Sin embargo, como se plantea a partir del modelo conceptual, mejorar los tiempos de espera y la disponibilidad de exámenes y especialistas, pueden reforzar la asistencia de usuarios a los servicios de urgencias de manera que la mejora deseada en los tiempos y personas esperando, no se consigue.

En este sentido, este trabajo plantea una comprensión sistémica de las dinámicas que se asocian con la entrada de pacientes a urgencias, que en espera de ser atendidas, generan sobrecarga de los servicios y altos tiempos de espera. Como se ha planteado desde otros trabajos como el de Hoot y Aronsky ${ }^{24}$ y el de Arkun et al. ${ }^{33}$, puede que muchos de estos usuarios sean no urgentes y auto referidos, quienes presentan sintomatologías que pueden ser solucionadas por otros servicios de menor complejidad y menor costo, tal como afirman Kraaijvanger et al. ${ }^{34} \mathrm{~A}$ partir de un análisis cuantitativo de los factores que tienen en cuenta las personas para decir asistir a urgencias en Bogotá, se plantea un modelo conceptual que espera enriquecer la discusión acerca de la sobrecarga de urgencias en la ciudad, mostrando la importancia de estudiar e intervenir las entradas al sistema y no sólo los procesos que se llevan a cabo en su interior.

El alto número de visitas a las unidades de urgencias no solamente depende del crecimiento de la población sino de la preferencia por asistir a este servicio ante cualquier enfermedad. En la úl- tima encuesta de evaluación de los servicios de las EPS a nivel nacional se observa que para Bogotá, el $84 \%$ de las personas que utilizaron en los últimos meses el servicio de urgencias, lo hizo pues sintió que su estado de salud era grave o podía tener consecuencias graves ${ }^{35}$. Estos resultados coinciden con los obtenidos por Lobachova et al. ${ }^{36}$ en su investigación. Sin embargo, faltan estudios e información sobre la realidad en los triage de las personas que llegan diariamente a urgencias.

En este sentido, nuestros resultados muestran que los usuarios que acuden a urgencias pertenecen principalmente a los estratos bajos y en general mantienen altos niveles de confianza en los médicos y en la eficacia de las unidades de urgencias. Es de destacar que en la medida en que los UBAUPA generen confianza en los usuarios, reducen la probabilidad de que los usuarios acudan directamente a urgencias, y viceversa. Adicionalmente, se planea que los UBA-UPA mejoren su capacidad para disminuir los tiempos de atención, ya que como lo soporta el estudio de López ${ }^{37}$, quien entrevistó a usuarios de urgencias con triage III o más, estos decidieron ir allí por las demoras para conseguir una cita en otros servicios (39.3\%). Lo que se expone en el modelo conceptual, es que las mejoras en los servicios de urgencias están generando un efecto contraproducente, pues se vuelve más atractivo para los pacientes acudir a estos ante cualquier enfermedad. Lo anterior no quiere decir que no se deban mejorar los centros de urgencias, sino que deben mejorarse también los centros donde se da atención prioritaria, de tal forma que sea más atractivo acudir allí que a urgencias.

El análisis cuantitativo evidenció que los usuarios de clase media pertenecientes al régimen contributivo acuden directamente a urgencias en busca de medicamentos y exámenes más que por obtener un servicio económico. Estos resultados coinciden con los obtenidos por Kraaijvanger et al. ${ }^{34}$, quienes afirman que los pacientes autoreferridos buscan una solución rápida a su patología y el acceso a exámenes. Sin embargo, este motivo no es compartido por todos los usuarios, pues el costo del servicio es un criterio de decisión más importante para los usuarios de estratos bajos. Esto indica que si los UBA-UPA aumentaran la facilidad para acceder a tratamiento y exámenes manteniendo el cobro por estos servicios, los usuarios de clase media tenderían a acudir en primera instancia a estos centros. En este sentido Rutten et al. ${ }^{38}$ afirma que para el caso de Holanda, ha resultado muy útil la incorporación de Cooperativas de Médicos Generales que ofrecen diag- 
nóstico y tratamiento como instancia previa a ser referido a una urgencia.

A partir de los datos primarios, se evidenció que los tiempos de espera en urgencias es un criterio relevante para asistir a estos centros sólo para los pacientes pediátricos. Esto puede estar relacionado con la percepción que, de acuerdo a la encuesta del Ministerio de Salud ${ }^{35}$, tienen los usuarios de que los tiempos de espera no son altos, pese a que Bustos et al. ${ }^{10}$ encontraron que en el 39\% de los casos los tiempos de espera en urgencias superan las 6 horas. En el caso de los pacientes pediátricos, este estudio encontró que estos dan mayor relevancia al criterio de bajo tiempo de espera, para asistir a urgencias. Dado esto, se propone que los UBA-UPA tengan una segregación formal para este tipo de usuarios con el fin de incentivar el que vayan primero a estos centros antes de acudir a urgencias.

Finalmente, este trabajo plantea que las políticas para disminuir la saturación de los servicios de urgencias deben basarse en la comprensión de los motivos y criterios que valoran los usuarios para acudir a los centros de urgencias. Esto con el fin de generar respuestas tempranas y eficientes que den un parte de tranquilidad al usuario ante su preocupación y de esta forma promuevan la confianza en los mecanismos alternos que se han diseñado.

\section{Colaboradores}

JCM Piñero participó en la concepción, investigación, recogida de información, análisis de la información y redacción del manuscrito. A Díaz igualmente participó en la concepción, investigación, análisis de la información y redacción del manuscrito. JS Martínez colaboró en la concepción, investigación, recogida de información, análisis de la información y redacción del manuscrito.

\section{Agradecimientos}

Este trabajo forma parte del proyecto titulado "Propuestas de mejora para el sistema general de seguridad social en salud", financiado por la Universidad Sergio Arboleda.

\section{Referencias}

1. Tudela P, Mòdol JM. La saturación en los servicios de urgencias hospitalarios. Emergencias 2015; 27(2):113120.

2. Savigny D, Adam T, compiladores. Aplicación del pensamiento sistémico al fortalecimiento de los sistemas de salud. Ginebra: Organización Mundial de la Salud; 2009.

3. Cooke DL, Yang H, Roger P, Rohleder TR, Lee RC, Strong D. Introducing System Dynamics Modeling to Health Care in Alberta. En: 25 International Conference of the System Dynamics Society. Boston; 2007. p.1-17.

4. Rohleder TR, Bischak DP, Baskin LB. Modeling patient service centers with simulation and system dynamics. Health Care Manag Sci 2007; 10(1):1-12.

5. Lattimer V, Brailsford S, Turnbull J, Tarnaras P, Simith $\mathrm{H}$, George S, Gerard K, Maslin-Prothero S. Reviewing emergency care systems I: insights from system dynamics modelling. Emerg Med J 2004; 21(6):685-691.

6. Fone D, Hollinghurst S, Temple M, Round A, Lester N, Weightman A, Roberts K, Coyle E, Bevan G, Palmer $\mathrm{S}$. Systematic review of the use and value of computer simulation modelling in population health and health care delivery. J Public Health Med 2003; 25(4):325-335.

7. Brailsford SC, Lattimer VA, Tarnaras P, Turnbull JC. Emergency and on-demand health care: modelling a large complex system. J Oper Res Soc 2004; 55(1):3442.

8. Restrepo Zea JH, Jaén Posada JS, Espinal Piedrahita JJ, Zapata Flórez PA. Saturación en los servicios de urgencias: Análisis de cuatro hospitales de Medellín y simulación de estrategias. Gerenc y Políticas Salud. 2018;17(34):1-15.

9. Ackoff RL. Systems thinking and thinking systems. Syst Dyn Rev 1994; 10(2-3):175-188.

10. Bustos Y, Castro J, Wen LS, Sullivan AF, Chen DK, Camargo CA. Emergency department characteristics and capabilities in Bogotá, Colombia. Int J Emerg Med 2015; 8(1):1-8 
11. Congreso de la República de Colombia. Ley N ${ }^{\circ} 100$ de diciembre de 1993. Por la cual se crea el sistema de seguridad social integral y se dictan otras disposiciones. [citado 2018 Jun 13]. Disponible en: http://achc.org. co/wp-content/uploads/2017/08/LEY-No-100-DEL1993-CREA-EL-SISTEMA-DE-SEGURIDAD-SOCIAL-INTEGRAL-Y-SE-DICTAN-OTRAS-DISPOSICIONES.pdf

12. Uribe-Gómez M. Nuevos cambios, viejos esquemas: las políticas de salud en México y Colombia en los años 2000. Cad Saude Publica 2017; 33(Supl. 2).

13. Camero Nader LM, Lagos Santamaría A, Triana Parga $\mathrm{R}$, et al. Fuentes de financiación y usos de los recursos del sistema general de Seguridad Social en Salud - SGSSS. Bogotá: Ministerio de Salud y Protección Social; 2016. Disponible en: https:/www.minsalud.gov.co/sites/ rid/Lists/BibliotecaDigital/RIDE/VP/FS/fuentes-y-usos-de-recursos-del-sgsss.pdf

14. Colombia. Secretaría Distrital de Salud de Bogotá. Emergencia Sanitaria [Internet]; 2016. [citado 2018 Jun 20]. Disponible en: http://www.saludcapital.gov. co/Paginas2/EmergenciaSanitaria2016.aspx

15. Colombia. Ministerio de salud y Protección Social. Resolución 005596 de diciembre de 2015. Por la cual se definen los criterios técnicos para el Sistema de Selección $y$ Clasificación de pacientes en los servicios de urgencias «Triage». [citado 2018 Jul 25]. Disponible en: https:// www.minsalud.gov.co/Normatividad_Nuevo/Resolución 5596 de 2015.pdf

16. Morales JC, Aranda JM, Cala S. Propuesta de una solución tecnológica como alternativa a los problemas de saturación en salas de urgencias de Bogotá, Colombia. Rev Cuba Inf en ciencias la salud 2017; 28(2):1-11.

17. Benach J, Tarafa G, Muntaner C. El copago sanitario y la desigualdad: ciencia y política. Gac Sanit 2012; 26(1):80-82.

18. Garcia-Subirats I, Vargas I, Mogollón-Pérez A, Paepe P, Silva MR, Unger JP, Vázquez ML. Barriers in access to healthcare in countries with different health systems. A cross-sectional study in municipalities of central Colombia and north-eastern Brazil. Soc Sci Med 2014; 106:204-213.

19. Van der Linden MC. Emergency department crowding: factors influencing flow. Amsterdam: University of Amsterdam; 2015.

20. Higginson I. Emergency department crowding. Emerg Med J 2012; 29(6):437-443.

21. Giunta DH, Pedretti AS, Elizondo CM, Ratti MFG, Quiros FGB, Waisman GD, Peroni HJ, Martínez B. Descripción de las características del fenómeno Crowding en la Central de Emergencia de Adultos, en un hospital universitario de alta complejidad: estudio de cohorte retrospectiva. Rev Med Chil 2017; 145(5):557-563.

22. Rodríguez-Páez FG, Jiménez-Barbosa WG, PalenciaSánchez F. Uso de los servicios de urgencias en Bogotá, Colombia: un análisis desde el triaje. Univ y Salud 2018; 20(3):215.

23. Durand A-C, Gentile S, Devictor B, Palazzolo S, Vignally P, Gerbeaux P, Sambuc R. ED patients: how nonurgent are they? Systematic review of the emergency medicine literature. Am J Emerg Med 2011; 29(3):333345.

24. Hoot NR, Aronsky D. Systematic review of emergency department crowding: causes, effects, and solutions. Ann Emerg Med 2008; 52(2):126-136.
25. Afilalo J, Marinovich A, Afilalo M, Colacone A, Léger $R$, Unger $B$, Giguère $C$. Nonurgent emergency department patient characteristics and barriers to primary care. Acad Emerg Med 2004; 11(12): 1302-10.

26. Gutiérrez FM, Gutiérrez FRM, Grisales CME, Vargas MAG, Molina PAS, Venegas LCM. Motivos de uso de los servicios de urgencias. Acta Odontológica Colomb 2016; 6(1):123-136.

27. Braun T, García Castrillo-Riesgo L, Kraffta T, Díaz-Regañón Vilchesb G. Frecuentación del servicio de urgencias y factores sociodemográficos. Gac Sanit 2002; 16(2):139-144.

28. Salud Colombia. I.P.S. Nivel III [Internet]. Bogotá: Salud Colombia; 2016. [citado 2018 Ene 15]. Disponible en: http://www.saludcolombia.com/actual/instituc/ins il11g.htm

29. Colombia. Secretaría Distrital de Planeación. Proyecciones de población por localidad para Bogotá 20162020. Bogotá: Secretaría Distrital de Planeación; 2014.

30. Sterman J. Business dynamics: systems thinking and modeling for a complex world. Massachusetts: Irwin/ McGraw-Hill; 2000.

31. Higginson I. Emergency department crowding. Emerg Med J 2012; 29(6):437-443.

32. Vermaak H. Working interactively with causal loop diagrams: intervention choices and paradoxes in practical applications. En: Boonstra J, De Caluwé L, editors. Intervening and changing: looking for meaning in interactions. Amersfoort: John Wiley \& Sons; 2007. p. 175-194

33. Arkun A, Briggs WM, Patel S, Datillo PA, Bove J, Birkhahn RH. Emergency department crowding: factors influencing flow. West J Emerg Med 2010; 11(1):10-15.

34. Kraaijvanger N, Rijpsma D, van Leeuwen H, Edwards M. Self-referrals in the emergency department: reasons why patients attend the emergency department without consulting a general practitioner first - a questionnaire study. Int J Emerg Med 2015; 8(1):1-6.

35. Colombia. Ministerio de Salud y Protección Social. Encuesta de evaluación de los servicios de las EPS - 2017. Bogotá: Ministerio de Salud y Protección Social; 2017.

36. Lobachova L, Brown DFM, Sinclair J, Chang Y, Thielker KZ, Nagurney JT. Patient and provider perceptions of why patients seek care in emergency departments. J Emerg Med 2014; 46(1):104-112.

37. López JA. Análisis comparativo de los servicios de urgencias 2008-2010. Hospitalaria 2011; (77):4-19.

38. Rutten M, Vrielink F, Smits M, Giesen P. Patient and care characteristics of self-referrals treated by the general practitioner cooperative at emergency-care-access-points in the Netherlands. BMC Fam Pract 2017; $18(1)$.

Artículo presentado en 08/02/2019

Aprobado en 18/11/2019

Versión final presentada en 20/11/2019

Editores jefes: Romeu Gomes, Antônio Augusto Moura da Silva 